\title{
A generic marginal value function for natural areas
}

\author{
Mark J. Koetse ${ }^{1}$ - Erik T. Verhoef ${ }^{2,3}$. $^{2}$ \\ Luke M. Brander ${ }^{1,4}$
}

Received: 21 August 2015 / Accepted: 18 October 2016 / Published online: 2 November 2016 C The Author(s) 2016. This article is published with open access at Springerlink.com

\begin{abstract}
We conduct a CV and a CE experiment using a generic rather than a situation-specific study design in order to obtain a generic marginal value function for different types of natural areas with different characteristics in the Netherlands. We develop a modelling approach in which we use CV and CE choice data in one model. The value function obtained shows that people attach value to the presence of natural areas, and that these values vary due to differences in the magnitude of areas, in distance to areas, and differences in accessibility and fragmentation of natural areas. We discuss how the value function can be used to incorporate potential substitution between natural areas. We also show that for approximately one-fifth of our sample there is no added value having any kind of natural area nearby and that people living in small municipalities tend to be indifferent between accessible and inaccessible areas. Our approach produces a generic marginal value function, which may be used to inform cost-benefit analyses and land use planning decisions that wish to incorpo-
\end{abstract}

$\bowtie \quad$ Mark J. Koetse

mark.koetse@vu.nl

Erik T. Verhoef

e.t.verhoef@vu.nl

Luke M. Brander

1.m.brander@vu.nl

1 Institute for Environmental Studies (IVM), Vrije Universiteit, De Boelelaan 1087, $1081 \mathrm{HV}$, Amsterdam, The Netherlands

2 Department of Spatial Economics, Vrije Universiteit, De Boelelaan 1105, 1081 HV, Amsterdam, The Netherlands

3 Tinbergen Institute, Amsterdam, The Netherlands

4 Brander Environmental Economics, 2408 Block F, 9-11 Hong Shing Street, Kornhill, Quarry Bay, Hong Kong 
rate positive and negative welfare externalities related to changes in the provision of natural areas. However, we also show that applying this value function to the regional or local scale may produce biased value estimates.

\section{JEL Classification H40 - Q26 · Q51}

\section{Introduction}

Natural areas and associated ecosystems provide a number of valuable services to society, such as recreational opportunities, aesthetic enjoyment, and environmental and agricultural services. Many of these ecosystem services have public good characteristics, and as a result they tend to be underprovided in the absence of policy intervention (e.g. Kotchen and Powers 2006; Smith et al. 2002). Political decision making regarding the provision of natural areas and land use planning requires information on ecosystem service values in order to make informed trade-offs against the (opportunity) costs of preservation. In this respect it is problematic that many of these ecosystem services, and especially recreation and aesthetic services, are generally not traded directly on markets, and their values are therefore not directly observed. The absence of information on these values has strong negative consequences for land use planning and likely leads to erroneous or at least uninformed planning decisions.

Numerous economic studies on the valuation of natural areas have therefore been conducted over the past 30 years. Useful summaries and meta-analyses of the valuation literature on forests are provided by Barrio and Loureiro (2010), Lindhjem (2007), Ojea et al. (2010), Zanderson and Tol (2009); and on wetlands by Brander et al. (2006, 2012), Brouwer et al. (1999), Ghermandi et al. (2010) and Woodward and Wui (2001). Descriptive overviews of the valuation literature on natural open space proximate to urban areas are provided in Fausold and Lilieholm (1999) and McConnell and Walls (2005). Brander and Koetse (2011) present meta-analyses on contingent valuation (CV) and hedonic pricing (HP) studies of peri-urban open space. With respect to the CV meta-analysis, the results show that the average value of a forest is around 1500 US\$ per hectare (in 2003 prices). Important factors influencing the value of open space are land use, services provided by the area, and size of the area. In particular, urban parks and areas used for recreation purposes have higher values, while size of the area decreases the value per hectare, which points to decreasing returns to size. The hedonic pricing meta-analysis results show that the average increase in house prices of being $100 \mathrm{~m}$ closer to open space is around $1 \%$. The results furthermore show that the impact of open space on house prices is substantially stronger when getting closer to open space (see also Dekkers and Koomen 2013).

Although the early valuation literature is dominated by contingent valuation and hedonic pricing studies, using choice experiments to elicit consumer preferences and estimate land use values has gradually become the state of the art. A fair amount of choice experiments on land use valuation have been conducted since the 1980s. There also is a growing number of choice experiment studies that aim to value natural areas and land use change (for recent contributions see, e.g. Bateman et al. 2009; Colombo et al. 2007; Colombo and Hanley 2008; Scarpa et al. 2007; Schaafsma et al. 2012, 
2013; Koetse and Brouwer 2015). A common feature of these studies is that they attempt to value changes to an existing natural area or ecosystem. The advantage of this form of application is that respondents who know the specified study site can make well-informed decisions about trade-offs between land use characteristics and monetary changes (often changes in local tax). A strong disadvantage of this sitespecific approach is that it lacks external validity (e.g. Bateman et al. 2006) because results are difficult to transfer to alternative sites, making the insights location specific. It is therefore difficult to generalise the results, thereby limiting the potential for value transfer and application to alternative policy assessments (e.g. Bateman et al. 2011; Navrud and Ready 2007).

In this study we therefore take a different approach and perform a choice experiment using a generic rather than a site-specific design. There are few studies that apply this approach. A notable exception is Viscusi et al. (2008), who use a generic choice experiment design to estimate citizens' willingness to pay for rivers and lakes with good water quality at the regional level in the USA. Our main contribution to the literature is that we obtain a marginal value function for natural areas that is more generic in nature and as such can be used in policy assessments that cover a larger geographic area than studies that use a site-specific study design. Related aims are to explicitly identify use and non-use values by analysing value differences between accessible and inaccessible areas, and to quantify the effects of fragmentation on the value of natural areas, an issue that is especially relevant for densely populated regions such as the Netherlands. The use of a generic setup, however, means that values for different types of natural areas obtained from the choice experiment are estimated relative to a reference category. In order to derive a value for this reference category we conduct a contingent valuation experiment. A noteworthy feature of our analysis is that we combine the choice data obtained from the $\mathrm{CV}$ and $\mathrm{CE}$ experiments in one model in order to obtain a generic marginal value function for natural areas in the Netherlands.

The remainder of this paper is organised as follows. In Sect. 2 we discuss the research design of the choice experiment and the contingent valuation study. Section 3 discusses the statistical design and the data collection procedure, while Sect. 4 presents the main estimation results of both the CV and CE study. In Sect. 5 we aim to uncover the main sources of preference heterogeneity, with a focus on use and non-use values and spatial heterogeneity. Section 6 concludes.

\section{Research design}

\subsection{Choice experiment design}

The purpose of the choice experiment is to assess consumer preferences for different types of natural areas and their characteristics, and for this we present respondents with choice alternatives that are of a generic nature and do not refer to a specific site or area. From the literature, it is evident that consumer preferences for natural areas may be affected by many characteristics (e.g. Van Zanten et al. 2014). The first attribute used to describe the study site is the type of area. We decided to include three wellknown and abundant types in the Netherlands, i.e., grassland, natural areas with water, 
and forests. ${ }^{1}$ In describing these three types, we used the descriptions developed in a previous study in which a large number of respondents in the Netherlands were asked to give their opinion on various types of natural areas (MNP 2007). From the metaanalysis by Brander and Koetse (2011), it is evident that size of and distance to natural areas have a substantial effect on associated values, so these two attributes were also included. The levels used for size are 2, 6, and $16 \mathrm{~km}^{2}$. These values are based on expert opinions on the size range of natural areas in the Netherlands. Levels used for distance to open space are 1,5 , and $15 \mathrm{~km} .^{2}$ In the description of this attribute, the road from a respondent's residence to the study site natural area is described as having a $60 \mathrm{~km} /$ hour speed limit, implying that it will take $15 \mathrm{~min}$ by car to get to the site in the case that the distance is $15 \mathrm{~km}$. The study by Brander and Koetse (2011) also shows that when an area is accessible for recreational use, its value increases considerably, and we therefore include an attribute for accessibility. In the Netherlands, natural areas are increasingly being fragmented by urban sprawl and transport infrastructure. In order to assess the negative externalities associated with these two types of fragmentation, we include both as attributes in the choice experiment. We implement fragmentation by urban sprawl by varying the representation of the natural area and distinguish between three degrees of fragmentation. When there is a single large area, there is no fragmentation, while medium and high degrees of fragmentation are represented by splitting the total area of the study site into two and four equal parts, respectively. ${ }^{3}$ The separate areas in the medium and high fragmentation situations are described as being approximately $500 \mathrm{~m}$ apart and connected by a single path that is suited for walking and biking only. With respect to fragmentation by transport infrastructure we also distinguish three levels, no fragmentation, medium fragmentation in which an area is fragmented by one road, and high fragmentation in which an area is fragmented by two roads. The two types of fragmentation are included in separate experiments that are otherwise identical, and for which the same sample of respondents is used. Finally, the payment vehicle used is a change in municipal tax, which is an annual tax in the Netherlands that is levied separately from national income taxes. Respondents are therefore very familiar with the tax. We use five levels of tax increases. A summary of attributes and attribute levels is provided in Table 1.

Since we keep the total landscape area constant and systematically vary the size of the natural areas, the size of natural areas and the size of the surrounding land uses are not independent. This may affect the results if respondents value positively or negatively the size of the surrounding land uses. The land uses surrounding the natural areas are described as consisting of mixed built-up area, and we ask the respondents to

\footnotetext{
${ }^{1}$ Small-scale grasslands are grasslands with a relatively small degree of openness because they contain many lines of trees and other types of vegetation (e.g. hedges). Natural areas with water usually contain several types of vegetation e.g. grass, trees and reed, and contain small rivers and pools of water. Large areas of water that can, for example, be used for sailing and other water-related activities, were explicitly excluded.

2 Around $60 \%$ of all trips made for recreational activities in nature in the Netherlands in 2006 and 2007 are trips with a one-way distance of less than or equal to $15 \mathrm{~km}$ (see CBS 2012).

${ }^{3}$ A study that uses a similar definition of fragmentation is Johnston et al. (2002). Their results show that households prefer unfragmented development and open space that is isolated from and not adjacent to residential development.
} 
Table 1 List of attributes and attribute levels in the choice experiment

\begin{tabular}{lll}
\hline Attributes & Number of levels & Levels \\
\hline Type of natural area & 3 & Small-scale grassland \\
& & Natural area with water \\
& Forest \\
Size of natural area & 3 & $2 \mathrm{~km}^{2}$ \\
& $6 \mathrm{~km}^{2}$ \\
& & $16 \mathrm{~km}^{2}$ \\
Distance to natural area & 3 & $1 \mathrm{~km}$ \\
& & $5 \mathrm{~km}$ \\
Fragmentation of natural area & 3 & $15 \mathrm{~km}$ \\
by urban sprawl & No fragmentation (1 patch) \\
Fragmentation of natural area & 3 & Medium fragmentation (2 patches) \\
by transport infrastructure & & High fragmentation (4 patches) \\
& & No fragmentation \\
Accessibility for recreation & & Medium fragmentation (one road) \\
Annual municipal tax & 2 & High fragmentation (two roads) \\
& & Accessible \\
& 5 & Not accessible \\
& & Increase of $€ 320,-$ \\
& & Increase of $€ 140,-$ \\
& & Increase of $€ 60,-$ \\
& & Increase of $€ 20,-$ \\
& & No change \\
\hline &
\end{tabular}

assume that the surroundings of their residential location, as represented in the choice card, resemble their current residential surroundings.

In attempting to value a good or service that is not offered on any real world market (or only in a very limited sense), it is important that respondents understand the good's essential features and characteristics. We therefore extensively describe the attributes and attribute levels, and additionally we use photographs to represent the three types of natural areas. We put much effort in ensuring that the photographs were sufficiently generic, in order to be able to generalise the results. We also made sure that the photographs did not contain 'alien' elements that may influence the results (e.g. buildings and animals). In presenting the choice options, we use text to describe the attribute levels, and below the text we include figures for graphical representation. These figures represent all attributes except for the payment vehicle. Furthermore, the size of areas and distances were all drawn to scale. Also note that we ensured that the total size of the natural area remained the same in situations with and without fragmentation; stated differently, size of the area, and degree of fragmentation are 
completely uncorrelated. Example choice cards are shown in Fig. 1 (for fragmentation by urban sprawl) and Fig. 2 (for fragmentation by infrastructure). ${ }^{4}$

We do not include a status quo alternative because we aim to identify generic relative preferences for natural areas and their characteristics in the Netherlands, rather than to identify whether and under what circumstances consumers prefer change to no change in a specific situation. Moreover, by leaving out the status quo we avoid status quo bias, and more generally prevent that unobserved characteristics or factors would affect the results. The absence of a status quo forces respondents to make a choice, which is exactly what we want given that we are interested in people's relative preferences for characteristics of natural areas. Even when a respondent would actually prefer the status quo to any of the choice options, the rational expectation is that he or she chooses the option that is least unattractive, thereby again revealing his or her relative preferences for natural areas. Crucial is that although the status quo usually provides the baseline level, (i.e., the baseline utility level for no change), this role is now fulfilled by the CV study. That is, the valuation of the reference area in the CE model is identical to the proposed change in the $\mathrm{CV}$ study, i.e., the $\mathrm{CV}$ study does incorporate a status quo option and is designed such that its replaces the status quo option in the CE.

\subsection{Contingent valuation design}

The purpose of the CV is to obtain a value for a natural area that is the omitted category of attribute levels in our choice model. In our choice experiment we distinguish between three types of natural areas, and we use small-scale grassland as the omitted category in the analysis since this is the most prevalent type of natural area in the Netherlands. The payment vehicle in the CV is the same as in the choice experiment, i.e. annual municipal tax. We initially chose to use a combination of a double-bounded dichotomous choice and an open-ended elicitation format. However, for estimation purposes we decided to only use the answer to the first bid, because the second bid lacks incentive compatibility (e.g. Whitehead 2002; Carson and Groves 2007). From here on, we will therefore treat the CV experiment as a single bounded dichotomous choice (SBDC) experiment. Respondents were first presented with the following text (translated from Dutch):

"Before presenting you with the 12 choice tasks, we like to know how much your household would be willing to pay for the presence of a natural area in the vicinity of your house. For this we ask you to imagine that in your current situation there is no natural area within a $15-\mathrm{km}$ radius from your house. Below we ask you to indicate how much your household would be willing to pay, through an increase in annual municipal tax, to change from this situation to a situation in which there is a small-scale grassland of 2 square kilometres at $1 \mathrm{~km}$ from your house, which is not fragmented and is accessible for recreation."

\footnotetext{
${ }^{4}$ Photographs used to represent the three types of natural areas and full descriptions of attributes are available upon request from the authors.
} 

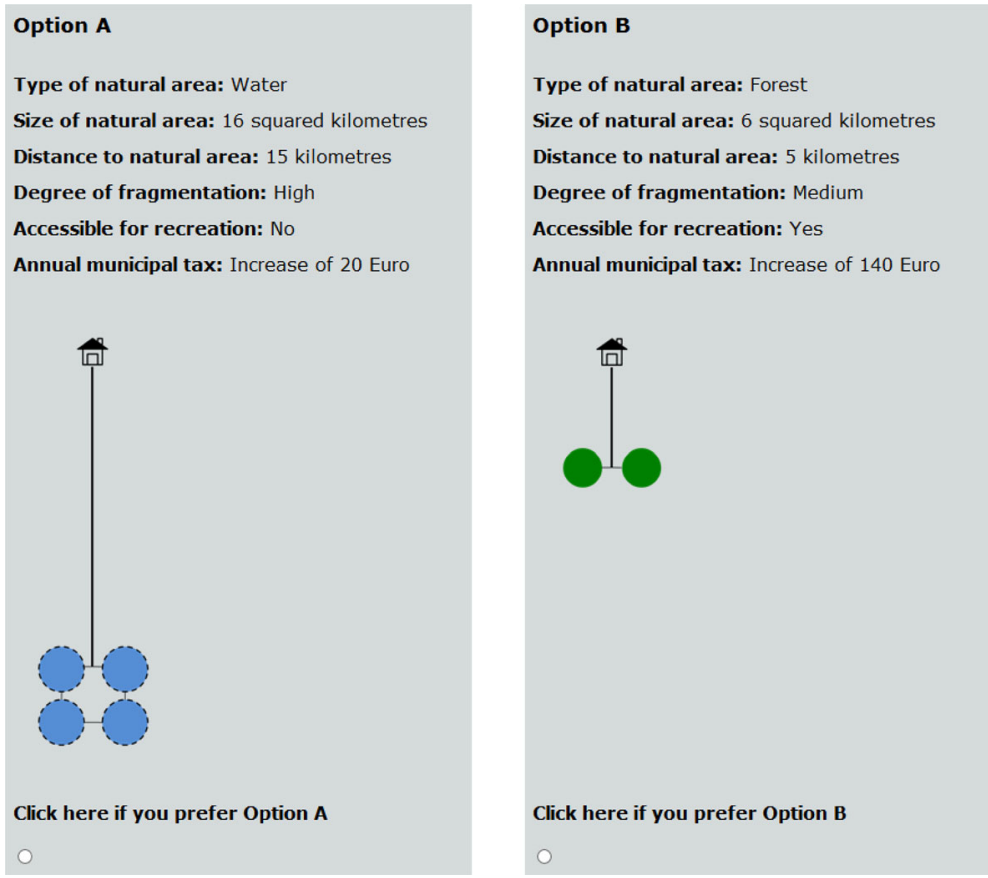

Fig. 1 Choice card example: fragmentation by urban sprawl
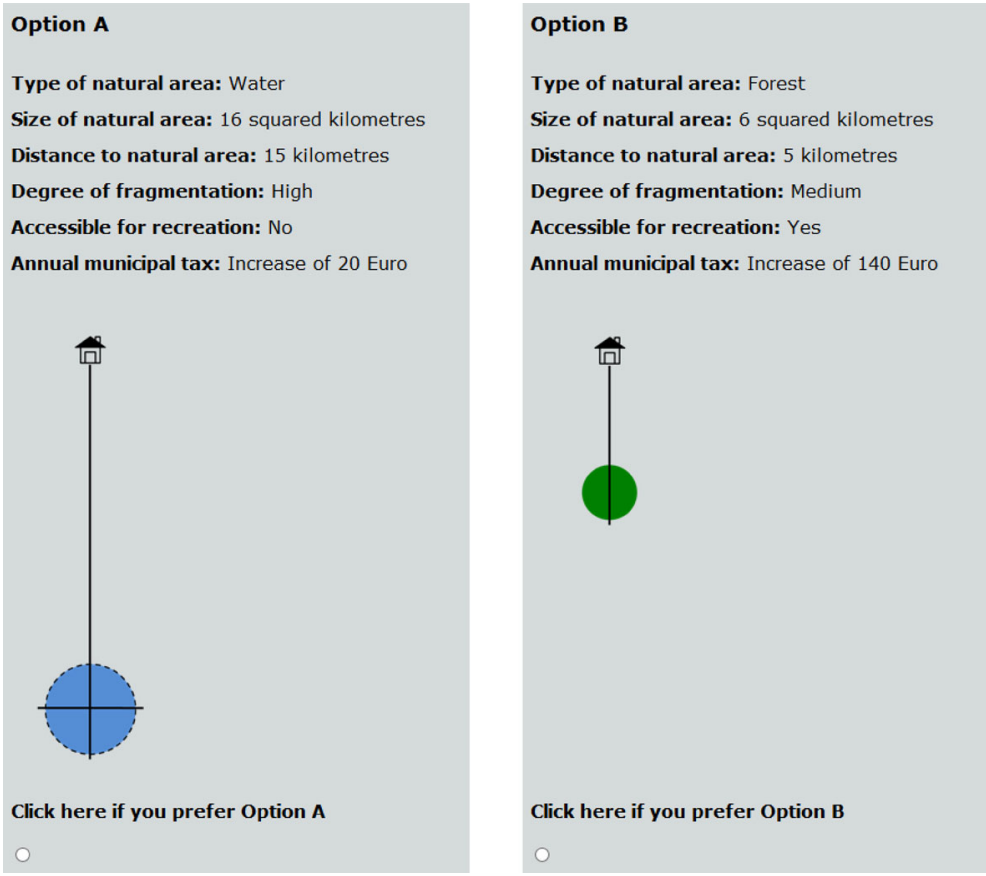

Fig. 2 Choice card example: fragmentation by infrastructure 
After this text the bid was provided, which respondents could either accept or reject. Obtaining insight into the optimal bid design is problematic in any bidding experiment. Various studies present first-best and second-best design rules (e.g. Alberini 1995; Cooper 1993; Cooper and Loomis 1992). However, all of them ultimately require knowledge on the actual WTP distribution, and few clear insights exist on creating an optimal design under distribution uncertainty. Also problematic in any bidding design is that respondents that are uncertain about their WTP tend to anchor their response to the initial bid provided (e.g. Herriges and Shogren 1996; Veronesi et al. 2011). For these reasons we decided to present respondents with a random bid drawn from a uniform distribution in the range 10-100 Euro, but with increments of 10 Euro. This way we cover a wide range of WTP values and can later on estimate a choice model with variation in the tax attribute.

\section{Statistical design and data collection}

The attributes and their levels can be combined to generate $(3 \times 3 \times 3 \times 3 \times 2 \times 5=)$ 810 possible choice alternatives. These obviously cannot all be shown to respondents; hence a fractional factorial statistical design was generated using the Sawtooth CBC software. The program uses a randomised design strategy, and produces a design that is nearly as orthogonal as possible within respondents (i.e. correlation between attribute levels for each respondent is minimal). The most often used design option in the program (complete enumeration) ensures that attribute levels are duplicated as little as possible within choice sets, a property called minimal overlap. This produces the most efficient design in terms of main effects. However, because we could guarantee a relatively large number of respondents a priori, we opted for a design method known as balanced overlap. This generates a slightly less efficient design because it allows for overlap of attribute levels in part of the choice tasks but has the advantage of obtaining better insight into potential interactions of attributes and attribute levels. We generate a statistical design containing 100 survey versions with a varying number of choice tasks. ${ }^{5}$ Before generating the statistical design we included some prohibitions for the monetary attribute, i.e. we prohibit differences in tax of 140, 260 and 300 Euro, because they do not add much information to the other possible tax differences. After generating the design we made one manual change. The accessibility attribute has two levels, and the initial design method is such that both levels are always included in a single choice task. This means that respondents always have to choose between a natural area that is accessible and one that is not. This is a property of the design that we found undesirable, because accessibility has been shown to be a very important factor in consumer preferences, and may dominate trade-offs between other attributes. In order to prevent this we adjust the accessibility levels such that $50 \%$ of the choice tasks contain the "accessible-not accessible" combination, $25 \%$ contain

\footnotetext{
5 The choice experiment also included choice tasks with decreases in tax to measure WTA. A single statistical design was generated with 12 choice tasks for each respondent, including both the choice tasks with tax increases and choice tasks with tax decreases. Therefore, each respondent did not by definition receive an equal number of choice tasks with tax increases.
} 
Table 2 Distribution of bid amounts chosen in the contingent valuation experiment

\begin{tabular}{lllllllllll}
\hline & \multicolumn{2}{l}{ Initial bid level } & & & & & & & & \\
\cline { 2 - 9 } & 10 & 20 & 30 & 40 & 50 & 60 & 70 & 80 & 90 & 100 \\
\hline \% Accept & 54.3 & 32.8 & 34.8 & 35.0 & 31.1 & 32.5 & 27.7 & 21.4 & 28.5 & 18.0 \\
Total \# of bids & 138 & 116 & 138 & 160 & 135 & 126 & 130 & 140 & 144 & 133 \\
\hline
\end{tabular}

the "accessible-accessible" combination, and $25 \%$ contain the "not accessible-not accessible" combination. In order to test the final statistical design we perform simulations using 1000 respondents; the results indicate that main effect standard errors for all main effects are more than acceptable. ${ }^{6}$ For a comparison of the different Sawtooth design strategies with other well-known design strategies we refer to Chrzan and Orme (2000).

After testing a first version of our questionnaire among colleagues from various disciplines, the questionnaire was tested among respondents that were sampled from a Dutch internet panel managed by TNS-NIPO, containing over 200,000 households. The panel is established through random sampling, meaning that each member of society has an equal chance to be added to the panel as long as he or she has conveyed the willingness to cooperate. Throughout the entire data collection process, respondents were sampled using representative sampling (for the entire Dutch population) on age, gender, education, household size and size of municipality. Results from this pretest $(N=188)$ revealed plausible signs and patterns for all attributes, so no adjustments to attribute levels were made. Pretest data were therefore also included in the full sample. Including the pretest, a total of 2100 questionnaires were sent out, and ultimately 1360 complete responses were obtained, implying a response rate of nearly $65 \%$. For the choice experiment, we have a total of 8334 observations.

For the CV experiment the share of bid rejections and acceptations is 68.3 and $31.7 \%$, respectively. Clearly, the majority of respondents rejected the bid, but a reasonable amount of respondents accepted. Acceptance percentages per bid level in the $\mathrm{CV}$ experiment are presented in Table 2. Acceptance rates go down for higher bid levels, with relatively steep decreases in acceptance rates at 20 Euro, 70 Euro and 100 Euro.

Statistics for the choice experiment show a plausible distribution of tax amounts chosen (see Table 3), with the smallest amount chosen most often and the largest amount chosen least often.

Background characteristics for our sample and for the entire population are presented in Table 4. The table shows that percentage shares on gender, age, household size and education for our sample are very comparable to those for the entire Dutch population. Distribution statistics show that people from municipalities with more than 100,000 inhabitants are underrepresented, which will turn out to have slight consequences for our average results that are presented in the next section. We do not have population statistics for recreation frequency, but statistics for our sample show that

\footnotetext{
${ }^{6}$ Full simulation results are available upon request from the authors.
} 
Table 3 Distribution of tax amounts chosen in the choice experiment

\begin{tabular}{llllll}
\hline & \multicolumn{6}{l}{ Tax amounts in choice experiment } \\
\cline { 2 - 6 } & $€ 0$ & $€ 20$ & $€ 60$ & $€ 140$ & $€ 320$ \\
\hline Percentage of respondents & 30.9 & 24.1 & 16.8 & 19.5 & 8.8 \\
\hline
\end{tabular}

the share of people that makes little or no use of natural areas for recreation purposes (i.e. once a year or less) is substantial. This turns out to be relevant as discussed later on in this paper.

Finally, various background factors may affect consumer preferences, and we obtained such data from various sources. From the owner of the panel of respondents used for this study (discussed later on), we obtained household size, gender, age, education, municipality and size of the municipality. By including additional questions in the survey we obtained information on frequency of visits to natural areas, household income and house ownership.

\section{Estimation results and value function}

The approach we took was to estimate a joint CV-CE model, in which the choices made in the $\mathrm{CV}$ experiment were added to the choice made in the $\mathrm{CE}{ }^{7}$ The specification of the choice model is then made such that the constant reveals the $\mathrm{CV}$ value estimate for grassland, and all other non-monetary coefficients can be used for deriving values for natural area attributes relative to the constant. With this approach the entire value function is obtained from a single model. We estimate a conditional logit (CL) model, in which all non-monetary attribute levels are dummy coded. ${ }^{8}$ We initially included just one tax attribute, but estimation results revealed a negative WTP for grassland in the CV experiment. This is not in line with results from a model with just the SBDC data, so we decided to include separate tax attributes for the CE and the CV experiment, both of which are included linearly. Although the inclusion of different coefficients for different monetary attributes in a choice model is usually inconsistent with the notion that such a coefficient reflects the (single-valued) marginal utility of income, it is permissible in this case because the $\mathrm{CV}$ experiment has its own exclusive parameters in the overall model, so that differences in the monetary attributes simply reflect differences in the scale of utility. After estimation we calculate CV WTP values based on the CV tax parameter, and CE WTP values based on the CE tax parameter. The results are presented in Table $5 .{ }^{9}$

\footnotetext{
7 We thank an anonymous reviewer for this suggestion.

8 All model estimations are conducted using Nlogit 5.0. For robustness we also estimated an RPL model using 500 Halton draws from a uniform distribution for each non-monetary attribute and fixed parameters for the tax attributes. Point estimates are very comparable to those from the CL model. For reasons of parsimony, we chose to use and present CL model results in this paper.

${ }^{9}$ We test the difference between tax coefficients using a WALD test and using the combinatorial test proposed by Poe et al. (2005, p. 359). These tests show that the tax coefficients are statistically different at a 7 and a $3 \%$ critical significance level, respectively.
} 
Table 4 Respondent background characteristics for sample and population

\begin{tabular}{|c|c|c|}
\hline Variable & Sample share (\%) 2009 & Population share $2009(\%)$ \\
\hline \multicolumn{3}{|l|}{ Gender } \\
\hline Male & 49.6 & 49.1 \\
\hline Female & 50.4 & 50.9 \\
\hline \multicolumn{3}{|l|}{ Age category } \\
\hline age $<25$ & 9 & 10.8 \\
\hline $25 \leq$ age $<35$ & 18 & 15.8 \\
\hline $35 \leq$ age $<45$ & 22 & 20.2 \\
\hline $45 \leq$ age $<55$ & 18 & 18.8 \\
\hline $55 \leq$ age $<65$ & 15 & 16.4 \\
\hline $65 \leq$ age & 18 & 18.1 \\
\hline \multicolumn{3}{|l|}{ Household size } \\
\hline 1 person & 18.4 & 20.3 \\
\hline 2 persons & 38.2 & 36.4 \\
\hline 3 persons & 16.0 & 16.3 \\
\hline 4 persons & 18.5 & 18.1 \\
\hline More than 4 persons & 9.0 & 8.9 \\
\hline \multicolumn{3}{|l|}{ Highest finished education } \\
\hline Primary school & 5.5 & 3.8 \\
\hline Secondary school (level 1 ; lowest) & 18.4 & 15.0 \\
\hline Secondary school (level 2) & 11.0 & 8.0 \\
\hline Secondary school (level 3) & 30.0 & 34.1 \\
\hline Secondary school (level 4; highest) & 9.2 & 7.1 \\
\hline Bachelor degree & 17.0 & 20.5 \\
\hline Master degree or higher $(\mathrm{PhD})$ & 7.4 & 10.6 \\
\hline Unknown & 1.5 & 0.9 \\
\hline \multicolumn{3}{|l|}{ Size of municipality } \\
\hline$>100,000$ people & 22.5 & 32.7 \\
\hline $50,000-100,000$ people & 24.6 & 18.3 \\
\hline $20,000-50,000$ people & 38.5 & 35.4 \\
\hline $10,000-20,000$ people & 11.9 & 11.5 \\
\hline$<10,000$ people & 2.6 & 2.2 \\
\hline \multicolumn{3}{|l|}{ Recreation frequency } \\
\hline Once a year or less & 17.7 & - \\
\hline Six times a year & 32.2 & - \\
\hline Once a month & 17.4 & - \\
\hline Twice a month & 15.4 & - \\
\hline Once a week & 12.8 & - \\
\hline More than once a week & 4.4 & - \\
\hline
\end{tabular}


Table 5 Conditional logit estimation results

\begin{tabular}{|c|c|c|c|c|}
\hline & \multicolumn{2}{|c|}{ CL coefficients } & \multirow[t]{2}{*}{ WTP } & \multirow[t]{2}{*}{ SE } \\
\hline & Beta & SE & & \\
\hline \multicolumn{5}{|l|}{ Type } \\
\hline Grass (CV constant) & -0.080 & 0.123 & $-€ 6.2$ & $€ 10.46$ \\
\hline Water (ref: grass) & $0.205^{* *}$ & 0.040 & $€ 22.7 * *$ & $€ 4.45$ \\
\hline Forest (ref: grass) & $0.630^{* *}$ & 0.042 & $€ 69.5^{* *}$ & $€ 4.72$ \\
\hline \multicolumn{5}{|l|}{ Size (ref: $\left.2 \mathrm{~km}^{2}\right)$} \\
\hline $6 \mathrm{~km}^{2}$ & $0.221 * *$ & 0.041 & $€ 24.3^{* *}$ & $€ 4.49$ \\
\hline $16 \mathrm{~km}^{2}$ & $0.302 * *$ & 0.041 & $€ 33.3^{* *}$ & $€ 4.57$ \\
\hline \multicolumn{5}{|l|}{ Distance (ref: $1 \mathrm{~km})$} \\
\hline $5 \mathrm{~km}$ & -0.052 & 0.040 & $-€ 5.7$ & $€ 4.41$ \\
\hline $15 \mathrm{~km}$ & $-0.344 * *$ & 0.041 & $-€ 38.0 * *$ & $€ 4.60$ \\
\hline \multicolumn{5}{|c|}{ Fragmentation 1 (ref: low $)^{\mathrm{a}}$} \\
\hline Medium & 0.029 & 0.059 & $€ 3.2$ & $€ 6.49$ \\
\hline Strong & $-0.237 * *$ & 0.062 & $-€ 26.2^{* *}$ & $€ 6.85$ \\
\hline \multicolumn{5}{|c|}{ Fragmentation 2 (ref: low) ${ }^{\mathrm{b}}$} \\
\hline Medium & $-0.156^{* *}$ & 0.054 & $-€ 17.2 * *$ & $€ 6.01$ \\
\hline Strong & $-0.515^{* *}$ & 0.057 & $-€ 56.9^{* *}$ & $€ 6.37$ \\
\hline \multicolumn{5}{|c|}{ Accessibility (ref: accessible) } \\
\hline Area not accessible & $-0.657 * *$ & 0.038 & $-€ 72.5 * *$ & $€ 4.29$ \\
\hline \multicolumn{5}{|l|}{ Tax (continuous) } \\
\hline Tax CV (in 100 Euro) & $-1.290 * *$ & 0.210 & - & - \\
\hline Tax CE (in 100 Euro) & $-0.906^{* *}$ & 0.024 & - & - \\
\hline NOBS & 9694 & & & \\
\hline $\log -\mathrm{L}$ & -5141 & & & \\
\hline Pseudo- $R^{2}$ (adjusted) & 0.233 & & & \\
\hline
\end{tabular}

\footnotetext{
${ }^{\text {a }}$ Fragmentation 1 is fragmentation by urban sprawl

${ }^{\mathrm{b}}$ Fragmentation 2 is fragmentation by transportation infrastructure $* *, *=$ Statistically significant at 1 and $5 \%$, respectively
}

The model performs well with an adjusted pseudo- $R^{2}$ of around 0.23 . Most attributes have the expected signs and are statistically significant a $5 \%$ critical significance level. The small and statistically insignificant grassland constant reveals that people are indifferent between having a grassland nearby or not (assuming the grassland is $2 \mathrm{~km}^{2}$, is at $1 \mathrm{~km}$ distance, is not fragmented and is accessible). ${ }^{10}$ The associated WTP is also small and statistically insignificant. It is further interesting to observe that natural areas with water and especially forests are valued substantially higher than grassland, which is also in line with findings from the literature (e.g. Earn-

\footnotetext{
10 We checked whether size of the municipality affects choices made in the CV experiment, and it does not, so no correction for underrepresentation of large municipalities (see Sect. 3 ) is necessary.
} 
hart 2001). The size of an area increases WTP (e.g. Brander and Koetse 2011) and our results show that there are strong decreasing returns to size. Specifically, an increase in size from 6 to $16 \mathrm{~km}^{2}$ has less added value per $\mathrm{km}^{2}$ than from 2 to $6 \mathrm{~km}^{2}$. ${ }^{11}$ We also find that the farther away a natural area is from the place of residence, the lower its value. The effect is close to linear, at least in the range used in the choice experiment. This is one of the few studies that explicitly incorporates the effects of fragmentation on preferences. The results show that although the effects of medium levels of fragmentation by urban sprawl and transportation are small, high levels of fragmentation are associated with substantial decreases in value. Fragmentation by transport infrastructure is considered to be worse than fragmentation by urban sprawl, presumably due to transport infrastructure being associated with more noise and air pollution and having a more serious barrier effect than residential areas. Finally, when an area is not accessible for recreation, its value decreases considerably. If we add the coefficients for inaccessibility to the associated coefficients for the different natural areas, negative coefficients result in all cases. This suggests that, on average, inaccessible areas have no value for consumers at all. We take a closer look at this issue in the next section.

The model performs well with an adjusted pseudo- $R^{2}$ of around 0.23 . Most attributes have the expected signs and are statistically significant a $5 \%$ critical significance level. The small and statistically insignificant grassland constant reveals that people are indifferent between having a grassland nearby or not (assuming the grassland is $2 \mathrm{~km}^{2}$, is at $1 \mathrm{~km}$ distance, is not fragmented and is accessible). ${ }^{12}$ The associated WTP is also small and statistically insignificant. It is further interesting to observe that natural areas with water and especially forests are valued substantially higher than grassland, which is also in line with findings from the literature (e.g. Earnhart 2001). The size of an area increases WTP (e.g. Brander and Koetse 2011) and our results show that there are strong decreasing returns to size. Specifically, an increase in size from 6 to $16 \mathrm{~km}^{2}$ has less added value per $\mathrm{km}^{2}$ than from 2 to $6 \mathrm{~km}^{2} .{ }^{13}$ We also find that the farther away a natural area is from the place of residence, the lower its value. The effect is close to linear, at least in the range used in the choice experiment. This is one of the few studies that explicitly incorporate the effects of fragmentation on preferences. The results show that although the effects of medium levels of fragmentation by urban sprawl and transportation are small, high levels of fragmentation are associated with substantial decreases in value. Fragmentation by transport infrastructure is considered to be worse than fragmentation by urban sprawl,

\footnotetext{
11 In the next section we will show that size of the municipality decreases the WTP for size of an area. Given that people from large municipalities are underrepresented in our sample, the average WTP values for size of an area therefore likely underestimates the population WTP. Based on estimates presented in the next section in Table 6 and distribution statistics presented in Table 4, we calculate that the magnitude of the underestimation is around $10 \%$.

12 We checked whether size of the municipality affects choices made in the CV experiment, and it does not, so no correction for underrepresentation of large municipalities (see Sect. 3) is necessary.

13 In the next section we will show that size of the municipality decreases the WTP for size of an area. Given that people from large municipalities are underrepresented in our sample, the average WTP values for size of an area therefore likely underestimates the population WTP. Based on estimates presented in the next section in Table 5 and distribution statistics presented in Table 2, we calculate that the magnitude of the underestimation is around $10 \%$.
} 
presumably due to transport infrastructure being associated with more noise and air pollution and having a more serious barrier effect than residential areas. Finally, when an area is not accessible for recreation its value decreases considerably. If we add the coefficients for inaccessibility to the associated coefficients for the different natural areas, negative coefficients result in all cases. This suggests that, on average, inaccessible areas have no value for consumers at all. We take a closer look at this issue in the next section.

In order to obtain absolute values for natural areas with various characteristics we combine the findings from the contingent valuation experiment and the choice experiment. More specifically, the $\mathrm{CV}$ result represents the value for the reference category in the $\mathrm{CE}$ model, so absolute values are obtained by computing changes from the mean $\mathrm{CV}$ value. Note that the mean $\mathrm{CV}$ value for grassland was small and statistically far from significant. For the value function, we therefore chose to put the WTP value for grassland at 0 Euro per household per year. With respect to the choice experiment we use the WTP estimates from Table 5; WTP estimates for size of an area are increased by $10 \%$ (see footnote 12). WTP estimates for size and distance are included per $\mathrm{km}^{2}$ and per $\mathrm{km}$, respectively. The generic marginal value function for natural areas in the Netherlands is then given by:

$$
\begin{aligned}
& \text { WTP }_{(\text {in Euro per household per year })}= \\
& \mathbf{I}(\text { grass }) \times € 0+\mathbf{I}(\text { water }) \times € 23+\mathbf{I}(\text { forest }) \times € 70+ \\
& \mathbf{I}\left(2<\text { size } \leq 6 \mathrm{~km}^{2}\right) \times\left(€ 6.7 \times\left(\text { size in } \mathrm{km}^{2}-2\right)\right)+ \\
& \mathbf{I}\left(\text { size }>6 \mathrm{~km}^{2}\right) \times\left(€ 27+€ 1 \times\left(\text { size in } \mathrm{km}^{2}-6\right)\right)+ \\
& \mathbf{I}(\text { distance }>5 \mathrm{~km}) \times(-€ 3.8 \times(\text { distance in } \mathrm{km}-5))+ \\
& \mathbf{I}(\text { fragmentation urban sprawl: high }) \times-€ 30+ \\
& \mathbf{I}(\text { fragmentation infrastructure: medium }) \times-€ 17+ \\
& \mathbf{I}(\text { fragmentation infrastructure: high }) \times-€ 57+ \\
& \mathbf{I}(\text { area not accessible }) \times-€ 73 .
\end{aligned}
$$

In equation (1) the $\mathbf{I}(*)$ represent indicators that are equal to one when the characteristic described between brackets holds for the natural area that is being studied. Note that filling in values for the different variables in the function may result in negative WTP values. In our $\mathrm{CV}$ and choice experiment, we did not explicitly include other types of land use and the potential for substitution between these different land use types, by which we would explicitly allow for a negative valuation of natural areas with certain characteristics. Still, negative values are possible, i.e. people may actually prefer to have other types of land use near their residential location instead of a natural area with certain characteristics. On the other hand, negative values may partly also be an artefact of the estimation, so treating them with caution is advisable.

\section{Spatial preference heterogeneity}

In this section we analyse potential heterogeneity of consumer preferences, with special attention to spatial heterogeneity. The main aim here is to test the applicability 
of the generic marginal value function in equation (1) to lower geographical scales (regional or local) than for which it was estimated (national). For this purpose we only use the choice experiment data. Although CV data may of course display substantial preference heterogeneity as well, the $\mathrm{CE}$ data reflect heterogeneity for a much wider range of attributes. In order to uncover sources of spatial preference heterogeneity, we estimate a CL model with interactions between choice attributes and respondents' background characteristics. To uncover spatial heterogeneity, we use size of the municipality as an indicator and distinguish between five size categories. In order to ensure that results are not caused by other factors, we also include potentially important background characteristics as covariates. We estimate a model with size of and distance to a natural area as linear choice attributes, ${ }^{14}$ and we model all choice attributes as linear functions of size of the municipality (four dummy variables; reference category is municipalities with $>100,000$ residents), two dummy variables on low recreation frequency (once a year; six times a year; reference category is more than six times a year) and two education dummy variables (education level medium: secondary school and bachelor's degree; education level high: master's degree or higher; reference category is education level lower than secondary school). In modelling the tax attribute, we also include monthly net income (centred around its mean and in 100 Euro) to test for possible income effects. ${ }^{15}$ After a first model estimation, we excluded some of the interactions between the attributes and the background variables, because they turned out to be small and/or statistically insignificant. The number of observations is slightly lower than for the attributes only model, due to missing observations in some of the interaction variables. Estimation results are presented in Table 6 and are robust to the excluded interactions.

The effect of income on the tax increase parameter is small and statistically insignificant. People with a low recreation frequency have lower sensitivity to differences in type and size of an area, distances to an area and to whether an area is accessible or not. Also they are more sensitive to tax increases. As a result, their overall WTP for natural areas and their characteristics is substantially lower. In short, people that make (almost) no use of natural areas care substantially less about the type and other characteristics of natural areas, and this group is relatively large at nearly $18 \%$ of the sample and population (see Table 4). Some caution is required in interpreting these results because recreation frequency may be endogenous. For example, recreation frequency may determine preferences for size of and distance to natural areas, but preferences for size and distance may also determine recreation frequency. Although

\footnotetext{
14 We include size and distance as linear attributes because or parsimony reasons only. Using dummy attribute coding for assessing heterogeneity would produce a substantial amount of additional interactions, but would have very little added value given the purpose of this section.

15 We asked respondents to fill in their monthly net income using nine income categories, and transformed this categorical variable to a continuous variable by taking the midpoint of each income group. Using this transformation the average monthly net income is equal to approximately $€ 2450$ Euro per month, while national average income in 2009 was equal to approximately $€ 2750$ per month (CBS 2013). The national average includes an allowance for holidays (approximately $8 \%$ ) and an end-of-year allowance, while we asked for the amount of money received monthly without taking these allowances into account. When adding these allowances to our average monthly net income, the difference is reduced to less than $€ 100$ Euro per month. Our sample therefore appears to be representative also in terms of income, and we therefore did not correct the estimated average CV estimate presented in the previous section.
} 
Table 6 Model including attributes modelled as linear functions of respondent background variables; dummy variables represented by $(\mathrm{d})$

\begin{tabular}{|c|c|c|}
\hline Attribute and interactions & Beta & SE \\
\hline \multicolumn{3}{|l|}{ Main attribute effects } \\
\hline Water (d) & $0.225 * * *$ & 0.056 \\
\hline Forest (d) & $0.704 * * *$ & 0.060 \\
\hline Size $\left(\right.$ in $\mathrm{km}^{2}$ ) & $0.028 * * *$ & 0.007 \\
\hline Distance (in km) & $-0.030 * * *$ & 0.005 \\
\hline Fragmentation 1 medium $(\mathrm{d})^{\mathrm{a}}$ & 0.028 & 0.059 \\
\hline Fragmentation 1 strong (d) & $-0.249 * * *$ & 0.063 \\
\hline Fragmentation 2 medium (d) ${ }^{b}$ & $-0.141 * * *$ & 0.054 \\
\hline Fragmentation 2 strong (d) & $-0.516^{* * *}$ & 0.057 \\
\hline Area not accessible (d) & $-0.814 * * *$ & 0.055 \\
\hline Tax increases (in 100 Euro) & $-0.883 * * *$ & 0.038 \\
\hline \multicolumn{3}{|l|}{ Interactions income } \\
\hline Tax increases $\times$ income & -0.0003 & 0.003 \\
\hline \multicolumn{3}{|l|}{ Interactions recreation frequency } \\
\hline Water $\times$ recreation frequency six times a year $(\mathrm{d})$ & -0.050 & 0.091 \\
\hline Water $\times$ recreation frequency once a year $(\mathrm{d})$ & -0.052 & 0.111 \\
\hline $\begin{array}{l}\text { Forest } \times \text { recreation frequency six times a year } \\
\text { (d) }\end{array}$ & -0.084 & 0.093 \\
\hline Forest $\times$ recreation frequency once a year $(\mathrm{d})$ & $-0.250^{* *}$ & 0.116 \\
\hline Size $\times$ recreation frequency six times a year $(d)$ & 0.006 & 0.007 \\
\hline Size $\times$ recreation frequency once a year $(\mathrm{d})$ & $-0.015^{*}$ & 0.008 \\
\hline Distance $\times$ recreation frequency six times a year $(\mathrm{d})$ & $0.015^{* *}$ & 0.007 \\
\hline Distance $\times$ recreation frequency once a year $(\mathrm{d})$ & $0.018 * *$ & 0.008 \\
\hline $\begin{array}{l}\text { Area not accessible } \times \text { recreation frequency six } \\
\text { times a year }(d)\end{array}$ & $0.218 * *$ & 0.086 \\
\hline $\begin{array}{l}\text { Area not accessible } \times \text { recreation frequency } \\
\text { once a year }(\mathrm{d})\end{array}$ & $0.404 * * *$ & 0.106 \\
\hline $\begin{array}{l}\text { Tax increases } \times \text { recreation frequency six } \\
\text { times a year }(d)\end{array}$ & -0.077 & 0.055 \\
\hline $\begin{array}{l}\text { Tax increases } \times \text { recreation frequency once a } \\
\text { year }(d)\end{array}$ & $-0.254 * * *$ & 0.075 \\
\hline \multicolumn{3}{|l|}{ Interactions education } \\
\hline Size $\times$ education medium $(\mathrm{d})$ & 0.010 & 0.007 \\
\hline Size $\times$ education high $(\mathrm{d})$ & $0.025^{* *}$ & 0.011 \\
\hline Distance $\times$ education medium $(\mathrm{d})$ & -0.009 & 0.007 \\
\hline Distance $\times$ education high $(\mathrm{d})$ & $-0.030^{* *}$ & 0.012 \\
\hline Tax increases $\times$ education medium $(\mathrm{d})$ & 0.051 & 0.056 \\
\hline Tax increases $\times$ education high $(d)$ & $0.250 * * *$ & 0.088 \\
\hline
\end{tabular}


Table 6 continued

\begin{tabular}{lll}
\hline Attribute and interactions & Beta & SE \\
\hline Interactions size of municipality & & \\
Size $\times$ municipality $50,000-100,000(d)$ & $-0.018^{* *}$ & 0.008 \\
Size $\times$ municipality $20,000-50,000(d)$ & $-0.017^{* *}$ & 0.008 \\
Size $\times$ municipality $10,000-20,000(d)$ & $-0.017^{*}$ & 0.010 \\
Size $\times$ municipality $<10,000(d)$ & -0.016 & 0.020 \\
Area not accessible $\times$ municipality $<10,000(d)$ & $0.462^{*}$ & 0.244 \\
\hline NOBS & 8334 & \\
Log-L & -4268 & \\
Pseudo $R^{2}$ (adjusted) & 0.258 & \\
\hline
\end{tabular}

a Fragmentation by urban sprawl

${ }^{\mathrm{b}}$ Fragmentation by transport infrastructure

$* * *, * *, *=$ Statistically significant at 1,5 and $10 \%$, respectively

both causal relationships suggest a strong correlation between preferences for nature and recreation frequency, endogeneity would imply that the coefficients on recreation frequency interactions are biased and are likely to be overestimates of the effects of recreation frequency on preferences for natural areas.

Education has substantial effects on preferences for the size of and distance to natural areas. This is not straightforward to explain but may reflect differences in the type and the duration of recreation between people with varying education levels. People with a higher education also have a lower tax increase coefficient, implying a higher WTP, ceteris paribus. The results therefore show that people with a higher level of education have substantially higher WTP for different types of natural areas, their size and their proximity.

Although the coefficients are not always statistically significant, the patterns for the effects of size of municipality show that the smaller the municipality of residence, the lower a person's sensitivity to size of an area. People living in more rural areas apparently appreciate small-scale natural areas more than people living in more urbanised areas. Also this result may be related to differences in type of recreation and in recreation frequency and duration. With respect to our search for use and non-use values an interesting finding is that people who live in (very) small municipalities appear to be substantially less sensitive to whether an area is accessible or not. The results do not provide evidence that on average these people actually prefer inaccessible areas over accessible areas, but do suggest that many people living in small municipalities and communities tend to be at least indifferent between accessible and inaccessible natural areas, without affecting their general valuation of these areas.

Clearly there is heterogeneity in preferences, and it is mainly correlated with education, recreation frequency and size of the municipality in which people live. The latter suggests that preferences vary across space and that applying the value function discussed in the previous section to lower geographical scales than the national level may 
produce large errors in value estimates. Using the value function for value transfer in order to obtain values at regional levels should therefore be done with caution, if at all.

\section{Conclusions and discussion}

Studies that attempt to put a monetary value on natural areas and their characteristics generally do so for a specific site or situation, making it difficult to generalise the results. We conduct a CV and CE study using a more generic study design in order to obtain marginal use and non-use values of different types of natural areas with different characteristics in the Netherlands. We develop a model in which we use the choice data obtained from the $\mathrm{CE}$ and $\mathrm{CV}$ experiment in a joint $\mathrm{CV}-\mathrm{CE}$ model, and find an average $\mathrm{CV}$ value for the vicinity of a small-scale grassland of 0 Euro per household per year. The results further show that natural areas with water and forests are preferred to grasslands. Increasing the size of an area and decreasing the distance between an area and the residential location have substantial positive effects on the derived WTP values. The results suggest that medium levels of fragmentation have limited consequences but that high levels of fragmentation have substantial detrimental effects on values of natural areas. The effect of fragmentation by transport infrastructure is somewhat stronger than the effect of fragmentation by urban sprawl. Also areas that are not accessible for recreation have substantially lower values, and it is likely that many respondents made their choices from a recreation perspective.

When analysing preference heterogeneity, the effects of recreation frequency, education, recreation frequency and size of the municipality stand out. Statistics show that approximately $20 \%$ of the population makes almost no use of natural areas for recreation purposes, and our results show that for this group there appears to be no added value having any kind of natural area nearby, regardless of its size, its type and whether it is accessible or not. Results further suggest that many people living in small municipalities tend to be at least indifferent between accessible and inaccessible natural areas, without affecting their general valuation of these areas. Probably a large part of this result may be explained by realising that most of these municipalities are located in generally 'green' regions or areas. This implies that although areas may not be accessible, people can still enjoy their aesthetic qualities because these areas are simply all around them. Of course, the heterogeneity found in this study has negative consequences for using the value function presented in Sect. 4 for value transfer to regional or local geographical scales. For value transfer to work at lower geographical scales, it is necessary to incorporate the main sources of (spatial) preference heterogeneity in the value function.

When combining the average $\mathrm{CV}$ and $\mathrm{CE}$ values it is clear that there is large variation in values of natural areas due to variations in size, variation in vicinity of areas to people and variation in accessibility and fragmentation of areas. The combined value estimates, or more generally the value function in Eq. (1), provide insight into generic marginal values of Dutch households for natural areas and their characteristics. Our approach and the value function obtained provide insight into the spatial variation of these values and into the average marginal value for an entire region or country, rather than for a specific site only. It therefore has relevance for policies and land use 
planning decisions that aim to account for positive and negative welfare effects related to the changes in the provision of natural areas.

An important next step is to transform the obtained marginal monetary values per household into marginal values for natural areas in the Netherlands. Conceptually a marginal value for a given natural area may be derived by filling in the value function for that area and for each household, and adding all household values. Of course, translation of the levels used in our study to actual practice may lead to practical difficulties. For example, the levels of fragmentation used in our choice experiment are rather crude compared to actual situations, so determining the level of fragmentation is largely a matter of judgement. Also we did not include all types of natural areas in our choice experiment, and so cannot assess values of excluded natural areas. Practical challenges aside, the values obtained would represent an estimate of the (average) marginal value of small-scale grasslands, natural areas with water and forests in the Netherlands. Of course, these values do not represent total economic values of natural areas, but only the values attached to them by citizens. Still, explicitly including these value estimates into welfare assessments may substantially improve the design of policies and land use planning decisions that directly or indirectly affect the stock and the quality of natural areas.

The most important potential drawback of our approach, as in any stated preference study, is the issue of hypothetical bias (e.g. Carson and Groves 2007; Vossler et al. 2012). In our CE there is little room for status quo bias, but forcing respondents to make a choice in the CE may affect value estimates. However, the impact of this approach on value estimates is unknown and potential bias may go either way. Future research in this area may focus on using a split sample design in which the effects of our approach are studied (e.g. by doing a treatment with a CVM and a forced choice in CE and compare it to a treatment with only a CE that includes an opt-out option). Potential bias in the CV experiment may be due to both protest statements (too many bid rejections) and giving socially desirable statements (too many bid acceptations). Although both issues may be important, their net effect is ambiguous. Future research may focus on assessing the relative magnitude of these effects.

Acknowledgements This study was supported through the programme 'Reinventing Landscape Planning in MetroLand,' funded by the Netherlands Organisation for Scientific Research (NWO), and from the ERA-Net BiodivERsA programme, with the national funder NWO, part of the 2011 BiodivERsA call for research proposals. We are indebted to Monique Brouwer from the 'Recreatie Noord-Holland' organisation for providing photographs of natural areas with water, Jules Bakker for Photoshop manipulation of most of the photographs used in the experiment, and Chris Jacobs (Vrije Universiteit, Amsterdam) for extensive JavaScript assistance.

Open Access This article is distributed under the terms of the Creative Commons Attribution 4.0 International License (http://creativecommons.org/licenses/by/4.0/), which permits unrestricted use, distribution, and reproduction in any medium, provided you give appropriate credit to the original author(s) and the source, provide a link to the Creative Commons license, and indicate if changes were made.

\section{References}

Alberini A (1995) Optimal designs for discrete choice contingent valuation surveys: single-bound, and bivariate models. J Environ Econo Manag 28:287-306 
Barrio M, Loureiro ML (2010) A meta-analysis of contingent valuation forest studies. Ecol Econ 69:10231030

Bateman IJ, Brouwer R, Ferrini S, Schaafsma M, Barton DN, Dubgaard A, Hasler B, Hime S, Liekens I, Navrud S, De Nocker L, Sceponaviciute R, Semeniene D (2011) Making benefit transfers work: deriving and testing principles for value transfers for similar and dissimilar sites using a case study of the non-market benefits of water quality improvements across Europe. Environ Resour Econ 50:365387

Bateman IJ, Day BH, Georgiou S, Lake I (2006) The aggregation of environmental benefit values: welfare measures, distance decay and total WTP. Ecol Econ 60:450-460

Bateman IJ, Day BH, Jones AP, Jude S (2009) Reducing gain-loss asymmetry: a virtual reality choice experiment valuing land use change. J Environ Econ Manag 58:106-118

Brander LM, Brauer I, Gerder H, Ghermandi A, Kuik O, Markandya A, Navrud S, Nunes PALD, Schaafsma M, Vos H, Wagtendonk A (2012) Using meta-analysis and GIS for value transfer and scaling up: valuing climate change induced losses of European wetlands. Environ Resour Econ 52:395-413

Brander LM, Florax RJGM, Vermaat JE (2006) The empirics of wetland valuation: a comprehensive summary and meta-analysis of the literature. Environ Resour Econ 33:223-250

Brander LM, Koetse MJ (2011) The value of urban open space: meta-analyses of contingent valuation and hedonic pricing results. J Environ Manag 92:2763-2773

Brouwer R, Langford IH, Bateman IJ, Turner RK (1999) A meta-analysis of wetland contingent valuation studies. Reg Environ Change 1:47-57

Carson RT, Groves T (2007) Incentive and informational properties of preference questions. Environ Resour Econ 37:181-210

CBS (2012) Statline. Accessed 26 april 2012, Den Haag, The Netherlands. http://statline.cbs.n1/StatWeb/ publication/?DM=SLEN\&PA=70220ENG\&D1=53-55,57-61,63-67,69,71,87-90, 102-103\&D2=41$44 \& \mathrm{D} 3=1 \& \mathrm{LA}=\mathrm{EN} \& H \mathrm{HR}=\mathrm{T} \& \mathrm{STB}=\mathrm{G} 1, \mathrm{G} 2 \& \mathrm{VW}=\mathrm{T}$

CBS (2013) Statline. Accessed 13 September 2013. Den Haag, The Netherlands. http://statline.cbs. $\mathrm{nl} /$ StatWeb/publication/?DMSLNL \&PA=70843NED\&D1=5\&D2=0-1,8-9, 15, 18\&D3=0-4\&D4=9\& $\mathrm{VW}=\mathrm{T}$

Chrzan K, Orme B (2000) An Overview and Comparison of Design Strategies for Choice-Based Conjoint Analysis Sawtooth Software Research Paper Series. http://www.sawtoothsoftware.com/support/techn ical-papers/design-of-conjoint-experiments/an-overview-and-comparison-of-design-strategies-forchoice-based-conjoint-analysis-2000. Sequiem, USA

Colombo S, Calatrava-Requena J, Hanley ND (2007) Testing choice experiment for benefit transfer with preference heterogeneity. Am J Agric Econ 89:135-151

Colombo S, Hanley ND (2008) How can we reduce the errors from benefits transfer? An investigation using the choice experiment method. Land Econ 84:128-147

Cooper JC (1993) Optimal bid selection for dichotomous choice contingent valuation surveys. J Environ Econ Manag 24:25-40

Cooper JC, Loomis JB (1992) Sensitivity of willingness-to-pay to bid design in dichotomous choice contingent valuation models. Land Econo 68:211-224

Dekkers JEC, Koomen E (2013) The monetary value of open space in urban areas: evidence from a dutch house price analysis. In: Van der Heide CM, Heijman WJM (eds) The economic value of landscapes. Routledge, New York, pp 245-260

Earnhart D (2001) Combining revealed and stated preference methods to value environmental amenities at residential locations. Land Econo 77:12-29

Fausold CJ, Lilieholm RJ (1999) The economic value of open space: a review and synthesis. Environ Manag 23:307-320

Ghermandi A, Van Den Bergh JCJM, Brander LM, De Groot HLF, Nunes PALD (2010) Values of natural and human-made wetlands: a meta-analysis. Water Resour Res 46:1-12

Herriges JA, Shogren JF (1996) Starting point bias in dichotomous choice valuation with follow-up questioning. J Environ Econ Manag 30:112-131

Johnston RJ, Swallow SK, Bauer DM (2002) Spatial factors and stated preference values for public goods: considerations for rural land use. Land Econ 78:481-500

Koetse MJ, Brouwer R (2015) Reference dependence effects on WTA and WTP value functions and their disparity. Environ Resour Econ. doi:10.1007/s10640-015-9920-2

Kotchen MJ, Powers SM (2006) Explaining the appearance and success of voter referenda for open-space conservation. J Environ Econ Manag 52:373-390 
Lindhjem H (2007) 20 years of stated preference valuation of non-timber benefits from Fennoscandian forests: a meta-analysis. J For Econ 12:251-277

McConnell V, Walls M (2005) The value of open space: evidence from studies of nonmarket benefits. Resources for the Future, Washington, D.C

MNP (2007) Belevingswaardemonitor 2006 Nota Ruimte (Perception Value Monitor 2006), Milieu-en Natuurplanbureau (MNP). Bilthoven, The Netherlands

Navrud S, Ready R (2007) Lessons learned for environmental value transfer. In: Navrud S, Ready R (eds) Environmental value transfer: issues and methods. Springer, Dordrecht

Ojea E, Nunes PALD, Loureiro ML (2010) Mapping biodiversity indicators and assessing biodiversity values in global forests. Environ Resour Econ 47:329-347

Poe GL, Giraud KL, Loomis JB (2005) Computational methods for measuring the difference of empirical distributions. Am J Agric Econ 87:353-365

Scarpa R, Campbell D, Hutchinson WG (2007) Benefit estimates for landscape improvements: sequential Bayesian design and respondents' rationality in a choice experiment. Land Econ 83:617-634

Schaafsma M, Brouwer R, Gilbert A, van den Bergh JCJM, Wagtendonk A (2013) Estimation of distancedecay functions to account for substitution and spatial heterogeneity in stated preference research. Land Econ 89:514-537

Schaafsma M, Brouwer R, Rose J (2012) Directional heterogeneity in WTP models for environmental valuation. Ecol Econ 79:21-31

Smith VK, Poulos C, Kim H (2002) Treating open space as an urban amenity. Resour Energ Econ 24:107129

Van Zanten BT, Verburg PH, Koetse MJ, Van Beukering PJH (2014) Preferences for European agrarian landscapes: a meta-analysis of case studies. Landsc Urban Plan 132:89-101

Veronesi M, Alberini A, Cooper JC (2011) Implications of bid design and willingness-to-pay distribution for starting point bias in double-bounded dichotomous choice contingent valuation surveys. Environ Resour Econ 49:199-215

Viscusi WK, Huber J, Bell J (2008) The economic value of water quality. Environ Resour Econ 41:169-187

Vossler CA, Doyon M, Rondeau D (2012) Truth in consequentiality: theory and field evidence on discrete choice experiments. Am Econ J Microecon 4:145-171

Whitehead JC (2002) Incentive incompatibility and starting-point bias in iterative valuation questions. Land Econ 78:285-297

Woodward RT, Wui YS (2001) The economic value of wetland sevices: a meta-analysis. Ecol Econ 37:257270

Zanderson M, Tol RSJ (2009) A meta-analysis of forest recreation values in Europe. J For Econ 15:109-130 